\title{
Mycoplasma genitalium: An Emerging Sexually Transmitted Infection
}

\author{
Jessian L. Munoz and Oluwatosin Jaiyeoba Goje \\ Ob/Gyn and Women's Health Institute, Cleveland Clinic, 9500 Euclid Avenue A81, Cleveland, OH, USA \\ Correspondence should be addressed to Oluwatosin Jaiyeoba Goje; jaiyeoo@ccf.org
}

Received 18 December 2015; Revised 2 February 2016; Accepted 8 February 2016

Academic Editor: Joaquim Ruiz

Copyright (C) 2016 J. L. Munoz and O. J. Goje. This is an open access article distributed under the Creative Commons Attribution License, which permits unrestricted use, distribution, and reproduction in any medium, provided the original work is properly cited.

\begin{abstract}
Mycoplasma genitalium has been recognized as a cause of male urethritis, and there is now evidence suggesting that it causes cervicitis and pelvic inflammatory disease in women. M. genitalium is a slow growing organism, and, with the advent of nucleic acid amplification test (NAAT), more studies are being performed, and knowledge about the pathogenicity of this organism elucidated. With NAAT detection, treatment modalities have been studied, and the next challenge is to determine the most effective antimicrobial therapy. Doxycycline, the first-line antibiotic for urethritis, is largely ineffective in the treatment of M. genitalium and furthermore, resistance to macrolide has also emerged. The most effective drug is Moxifloxacin although there are emerging reports of resistance to it in various parts of the world. This paper not only highlights the current research and knowledge, but also reviews the diversity of health implications on the health of men and women infected with M. genitalium. Alternate antibiotics and the impact of M. genitalium on infertility are areas that require more studies as we continue to research into this microorganism.
\end{abstract}

\section{Introduction}

Mycoplasma genitalium is a slow growing organism, and the advent of nucleic acid amplification test (NAAT) has shed more light on this emerging sexually transmitted infection (STI). There has been an increase in interest, research, and knowledge about $M$. genitalium in recent years. The most recent Centers for Disease Control and Prevention (CDC) sexually transmitted diseases treatment guidelines, published in 2015, discuss M. genitalium under emerging issues [1]. It is therefore imperative that both scientist and clinicians understand the organism, pathogenicity, and sequelae.

M. genitalium infection occurs globally, having been found in every country where it has been sought. The prevalence of $M$. genitalium varies within countries and communities. A large study in the Netherlands documented a $4.5 \%$ prevalence in their community, second only to Chlamydia trachomatis (8.3\%), and greater than Neisseria gonorrhoeae (1.3\%) and Trichomonas vaginalis (1.4\%) [2]. In Sweden, $6.3 \%$ of patients at a sexually transmitted diseases (STD) clinic were noted to be infected with $M$. genitalium, with $45 \%$ of these patients having partners who also harbored the pathogen [3]. In England, the prevalence of M. genitalium was considerably lower (1.2\%) [4]. A study from the United States showed a prevalence of $0.4 \%$ in young adults [5]. In that study, $M$ genitalium was more prevalent than $N$. gonorrhoeae but less prevalent than $C$. trachomatis, and it was strongly associated with sexual activity.

M. genitalium was detected in $5.8 \%$ of human immunodeficiency virus (HIV) positive men by PCR analysis in Brazil [6]. Mavedzenge et al. documented that it may be an independent risk factor for the acquisition of HIV-1 in Zimbabwe and Ugandan women [7]. In addition, $M$. genitalium was also associated as a cofactor for HIV infection in a case-control study of Ugandan women [8].

There exist many barriers and gaps to further understand M. genitalium infection and its impact on human health. Currently, there is no uniform method of detection of $M$. genitalium, which makes a collective comparison difficult. In addition, the precise role of $M$. genitalium in disease such as pelvic inflammatory disease, infertility, and pregnancy is not currently known, and more studies are needed. M. genitalium treatment has also shown to be difficult given mechanisms of resistance and variation in clinical management. These 
are gaps we highlight in this review and compare the data available to assess the importance of $M$. genitalium to disease processes and treatment. To analyze the literature with respect to $M$. genitalium in human pathology, we reviewed articles available on PubMed in the English language from 1981 to 2015, which explored M. genitalium as a human pathogen.

\section{Diagnostic Consideration}

A significant step in M. genitalium research would be uniform detection of acute infection and prior exposure. Isolation and culturing of $M$. genitalium is slow, time consuming, and not feasible when there is a need to institute immediate antimicrobial therapy. Therefore, NAAT is the preferred diagnostic method where feasible. Although research companies have quantitative PCR detection kits in the market, the United States Food and Drug Administration (FDA) has not approved any of these methods for the clinical screening or detection of $M$. genitalium. Vandepitte and colleagues [8] compared two commercially available kits (TIB MOLBIOL LightMix kit and the Diagenode $M$. genitalium real-time PCR kit) as well as an in-house PCR method using the Roche Diagnostics cobas z 480 analyzer [9]. TIB MOLBIOL LightMix kit targeted the $m g 219$ gene, Diagenode $M$. genitalium real-time PCR kit targeted the gap gene, and the in-house kit targeted the $\mathrm{MgPal}$ adhesion protein gene. The commercial kits had a sensitivity of $92.6 \%$ and $87 \%$, respectively, and a specificity of $100 \%$ which was concordant with the in-house kit that was $>95 \%$.

In an effort to establish a simpler and streamlined protocol for M. genitalium detection, Takanashi and colleagues developed a PCR test using InvaderPlus ${ }^{\circledR}$ technology, carrying out both the endonuclease and PCR in the same simple step [10]. This approach would require less genetic material and would be of less labor and would be time consuming. The approach was tested with first-void urine samples and the PCR target was the 16S rRNA gene of M. genitalium. The InvaderPlus assay was comparable to typical hybridization microtiter PCR, able to detect as few as 10 DNA copies per reaction. A 99.3\% concordance between the two assays was noted (137/138). While this assay was not tested with urethral, pharyngeal, vaginal, or anorectal swabs for clinical determination, the data was found to be promising.

Another opportunity for biotechnology in M. genitalium pathology is establishing genetic markers of resistance to first-line therapy. Currently, $M$. genitalium antibiotic therapy reflects $C$. trachomatis therapy (Doxycycline and Azithromycin). Resistance to azithromycin through mutations in ribosomal genes has been reported [11]. In France, a combination of PCR and FRET analysis revealed $14.2 \%$ of samples contained antibiotic-resistance associated mutations of the $23 r R N A$ gene, but no correlation with treatment was established [12]. By screening the $23 r R N A$ gene in Australia, it was noted that $20 \%$ of pretreatment and $100 \%$ of treatment failure samples contained mutations of this gene which may confer resistance to macrolides [13]. Therefore, $M$. genitalium may have intrinsic as well as induced mechanisms of resistance to antibiotic therapy.

\section{Clinical Considerations}

3.1. M. genitalium in Men. Although Neisseria gonorrhoeae and Chlamydia trachomatis are well known causes of male urethritis, M. genitalium has arisen as another cause. In 1981, M. genitalium was isolated for the first time from the samples of 2 men with nongonococcal urethritis (NGU) [14], and since then, data supporting the role of $M$. genitalium as a cause of male urethritis has increased over the years. Studies showed that M. genitalium infection had a 6.5 -fold increased risk of urethritis (22\% versus $4 \%$ of controls, 95\% CI $2.1-$ 19.5), after controlling for N. gonorrhoeae and C. trachomatis [15]. M. genitalium has also been implicated in balanitis and posthitis. In a study of 114 men with nongonococcal urethritis, M. genitalium significantly correlated with the development of balanitis and posthitis [16]. Conversely, sperm concentration was negatively correlated with $M$. genitalium infection [17].

There is conflicting data on M. genitalium and circumcision. A study on male circumcision in Kenya revealed M. genitalium was detected in $13.4 \%$ of uncircumcised men compared with $8.2 \%$ of circumcised men $(p=0.06)$. Adjusted odds ratio showed a $50 \%$ reduction in $M$. genitalium infection when men were circumcised [18]. On the other hand, a study from England, did not detect a relationship between $M$. genitalium and circumcision, although it was underpowered [19].

Trends in oral and anal sex have increased over the past decades; anal intercourse has doubled over a 10-year period [20]. A study of 1778 men screened by urine and anorectal swabs revealed 91 (5.1\%) were positive for M. genitalium. Of note, $71.4 \%$ of $M$. genitalium positive patients' in the study had positive anorectal swab. [21]. Another study using anal swabs reported $4.2 \% M$. genitalium positive screen among HIV positive men who have sex with men [22].

In addition, $24.3 \%$ of $M$. genitalium infected women were noted to have positive anorectal swabs [23]. M. genitalium has been found in the anorectal region but its pathogenicity in causing clinical proctitis has not been elucidated and more research is required.

3.2. M. genitalium in Women. The incidence of M. genitalium and $C$. trachomatis is relatively similar in high-risk women. In a cross sectional study at an STD clinic, M. genitalium and C. trachomatis were detected in $6 \%$ and $10 \%$ of women, respectively [24]. These women presented with cervicitis, and, in addition, $M$. genitalium was detected in 59\% of their male partners. The organism has also been implicated in Pelvic Inflammatory Disease (PID); M. genitalium was detected in $13 \%$ of PID patients and $0 \%$ of their controls [25]. It is important to note that the CDC recommended antibiotic regimens for PID, not effective against $M$. genitalium [26].

Although N. gonorrhoeae and C. trachomatis are known causes of mucopurulent cervicitis, a significant proportion of cervicitis are of unknown etiology [27]. Manhart and colleagues explored $M$. genitalium as a cause of mucopurulent cervicitis [28] and reported M. genitalium in $7.0 \%$ of 719 women. In addition, risk factors for M. genitalium cervicitis were determined, and they include younger age, multiple 
sexual partners, and a history of miscarriage, smoking, or douching. Thus it was concluded that M. genitalium might be a cause of mucopurulent cervicitis.

Furthermore, M. genitalium was present in $16 \%$ of endometrial biopsies performed in 58 women with histologically confirmed acute endometritis [28]. Known potential complications of endometritis include infertility, pelvic peritonitis, abscess formation, and sepsis.

Given that M. genitalium is associated with endometritis, cervicitis, and PID, M. genitalium may have a significant effect on reproductive health and pregnancy outcomes. Unlike C. trachomatis, some studies using PCR have shown a correlation with ectopic pregnancy but others using serological markers have not shown this relationship [29, 30]. In addition, $M$. genitalium infection does not correlate with early miscarriage [31] but has been associated with increased risk of preterm delivery. $M$. genitalium was associated with preterm labor in a study involving 667 Peruvian women who underwent preterm labor [32]. M. genitalium was not associated with any other risk factors of preterm labor [31]. Another study of 134 women from the United States revealed a preterm labor rate of $28 \%$ and $M$. genitalium was detected in $20.2 \%$ of these patients [33]. Taken together, these studies show a relationship between $M$. genitalium infection and the female physiology, pregnancy, and pathophysiology.

3.3. M. genitalium and Infertility. Approximately $9 \%$ of conceiving couples may experience infertility [34], while patency of fallopian tubes, disorders of ovulation, and sperm function are common, bacterial infections also play a role in infertility $[35,36]$. In vitro studies confirmed $M$. genitalium can bind to fallopian tube epithelium [37]. An Iranian study using PCR (16S rRNA) to detect $M$. genitalium found the pathogen in $2.8 \%$ cervical swabs of infertile women [38]. Interestingly, in another study, M. genitalium was detected in samples collected from both cervical swabs as well as abdominal laparoscopic washings of women with infertility [39]. The studies suggest $M$. genitalium might play a role in female infertility. Conversely, the possible role of $M$. genitalium in male fertility needs further research. A large meta-analysis which included the data from studies including 307 men with infertility, suggested a minimal role for $M$. genitalium in male infertility [40]. Thus, more research is required to establish a clear role between $M$. genitalium infection and infertility.

\section{Antibiotic Therapy}

The current treatment guidelines for NGU are oral Doxycycline $100 \mathrm{mg}$ twice a day for 1 week or single dose oral Azithromycin $1 \mathrm{~g}$. NGU may also be caused by M. genitalium and Doxycycline is ineffective in eradicating $M$. genitalium infection, with efficiency ranging from 17 to $90 \%$, irrespective of macrolide resistance [41]. In addition, with increasing resistance, azithromycin has become progressively less effective [42]. Resistance to macrolides has been reported as high as $30 \%-40 \%$ in certain populations [43]. Furthermore, to test efficacy, the establishment of a time period for test of cure (TOC) is required; the exact time to eradication of
M. genitalium after treatment is variable. One study showed 96\% eradication within 8 days after Azithromycin therapy, as measured by PCR [44]. Interestingly enough, these authors also noted those patients with resistant strains were detected after a period of negative tests, thus suggesting test of cure should be held until 3-4 weeks after treatment. When treated with Moxifloxacin, PCR testing was negative within a week. In this study by Falk and colleagues, doxycycline treatment did not eradicate $M$. genitalium in 6 of 8 patients in this study.

Extended azithromycin therapy has been suggested as an alternative method for antibiotic therapy and to reduce the development of resistant strains [45]. To explore the efficacy of this extended treatment 54 females and 31 males were treated with oral Azithromycin $500 \mathrm{mg}$ on day 1 and $250 \mathrm{mg}$ on the following 4 days; test of cure (TOC) analyses were performed 6 weeks later; $25 \%$ of patients still tested positive for M. genitalium at TOC [46]. The greater risk of treatment failure is the development of macrolide resistant strains in the community.

Attempts to explore the use of other antibiotics for $M$. genitalium eradication have been made difficult because $M$. genitalium lacks a cell wall thus; it is inherently resistant to antibiotics targeting cell wall synthesis such as beta-lactams and penicillins. Oral Moxifloxacin (dose of $400 \mathrm{mg}$ daily for 7-10 days), a member of the fluoroquinolone family, has shown significant efficiency in $M$. genitalium eradication of macrolide resistant strains. The drawbacks of Moxifloxacin usage are the broad spectrum, side effects, the cost in certain countries, and contraindication in pregnancy. In addition, mutations associated with fluoroquinolone resistance ( $\operatorname{parC}$ or gyrA) genes have been reported in $15 \%$ of patient samples prior to treatment [47]. Overall, in the management of $M$. genitalium urethritis and cervicitis, doxycycline has poor response compared to Azithromycin. Moxifloxacin has been found to be more effective in patients who have failed previous therapy. M. genitalium should be suspected in persistent or recurrent urethritis, cervicitis, and PID. Patients who fail the CDC recommended therapy for PID treatment should be treated using Moxifloxacin for 14 days, and where available, clinicians may test women who have failed recommended PID treatment for M. genitalium and treat them accordingly if positive [1].

\section{Conclusions}

Studies demonstrate that $M$. genitalium is an emerging sexually transmitted infection. Additional research is needed regarding pathogenicity and treatment, and there is a need for a standardized NAAT for clinical detection and resistance. Treatment for M. genitalium infection should be considered when patients fail first line treatment for urethritis, cervicitis, and PID. In the United States, most treatments will occur in the context of syndromic management of persistent or recurrent urethritis, cervicitis, and PID until we have an FDA approved diagnostic method. If $M$. genitalium diagnosis is performed in communities where NAAT testing is available, Azithromycin extended regimen (500 $\mathrm{mg}$ day 1, $250 \mathrm{mg}$ days 2-5) may be considered as first line treatment, but Moxifloxacin is the appropriate drug in patients with previous 
treatment failure. In addition, HIV positive patients should be treated with the same antimicrobial therapy regimen as HIV negative patients. Sex partners should be treated according to guidelines for patients with NGU, cervicitis, and PID [1].

\section{Conflict of Interests}

The authors declare that there is no conflict of interests regarding the publication of this paper.

\section{References}

[1] K. A. Workowski and G. A. Bolan, "Sexually transmitted diseases treatment guidelines, 2015," MMWR Recommendations and Reports, vol. 64, no. RR-03, pp. 1-137, 2015.

[2] A. S. de Jong, J. C. Rahamat-Langendoen, P. van Alphen et al., "Large two-centre study into the prevalence of Mycoplasma genitalium and Trichomonas vaginalis in the Netherlands," International Journal of STD \& AIDS, 2015.

[3] C. Anagrius, B. Loré, and J. S. Jensen, "Mycoplasma genitalium: prevalence, clinical significance, and transmission," Sexually Transmitted Infections, vol. 81, no. 6, pp. 458-462, 2005.

[4] P. Sonnenberg, C. A. Ison, S. Clifton et al., "Epidemiology of Mycoplasma genitalium in British men and women aged 1644 years: evidence from the third National Survey of Sexual Attitudes and Lifestyles (Natsal-3)," International Journal of Epidemiology, 2015.

[5] L. E. Manhart, K. K. Holmes, J. P. Hughes, L. S. Houston, and P. A. Totten, "Mycoplasma genitalium among young adults in the United States: an emerging sexually transmitted infection," American Journal of Public Health, vol. 97, no. 6, pp. 1118-1125, 2007.

[6] F. A. M. da Costa, R. C. da Silva, L. B. Arruda, P. Montanheiro, A. J. da Silva Duarte, and J. Casseb, "Prevalence of Mycoplasma genitalium among HIV-infected men in São Paulo city detected by realtime polymerase chain reaction," International Journal of STD and AIDS, vol. 21, no. 1, pp. 23-25, 2010.

[7] S. N. Mavedzenge, B. Van Der Pol, H. A. Weiss et al., "The association between Mycoplasma genitalium and HIV-1 acquisition in African women," AIDS, vol. 26, no. 5, pp. 617-624, 2012.

[8] J. Vandepitte, H. A. Weiss, J. Bukenya et al., "Association between Mycoplasma genitalium infection and HIV acquisition among female sex workers in Uganda: evidence from a nested case-control study," Sexually Transmitted Infections, vol. 90, no. 7, pp. 545-549, 2014.

[9] C. Le Roy, S. Pereyre, and C. Bébéar, "Evaluation of two commercial real-time PCR assays for detection of Mycoplasma genitalium in urogenital specimens," Journal of Clinical Microbiology, vol. 52, no. 3, pp. 971-973, 2014.

[10] M. Takanashi, S. Ito, H. Kaneto et al., "Development and clinical application of an InvaderPlus ${ }^{\circledR}$ assay for the detection of genital mycoplasmas," Journal of Infection and Chemotherapy, vol. 21, no. 7, pp. 516-519, 2015.

[11] M. Bissessor, S. N. Tabrizi, J. Twin et al., "Macrolide resistance and azithromycin failure in a Mycoplasma genitalium-infected cohort and response of azithromycin failures to alternative antibiotic regimens," Clinical Infectious Diseases, vol. 60, no. 8, pp. 1228-1236, 2015.

[12] A. Touati, O. Peuchant, J. S. Jensen, C. Bébéar, and S. Pereyrea, "Direct detection of macrolide resistance in Mycoplasma genitalium isolates from clinical specimens from France by use of real-time PCR and melting curve analysis," Journal of Clinical Microbiology, vol. 52, no. 5, pp. 1529-1555, 2014.

[13] J. Twin, J. S. Jensen, C. S. Bradshaw et al., "Transmission and selection of macrolide resistant mycoplasma genitalium infections detected by rapid high resolution melt analysis," PLoS ONE, vol. 7, no. 4, Article ID e35593, 2012.

[14] J. G. Tully, D. Taylor-Robinson, R. M. Cole, and D. Rose, "A newly discovered mycoplasma in the human urogenital tract," The Lancet, vol. 317, no. 8233, pp. 1288-1291, 1981.

[15] P. A. Totten, M. A. Schwartz, K. E. Sjöström et al., "Association of Mycoplasma genitalium with nongonococcal urethritis in heterosexual men," The Journal of Infectious Diseases, vol. 183, no. 2, pp. 269-276, 2001.

[16] P. J. Horner and D. Taylor-Robinson, "Association of Mycoplasma genitalium with balanoposthitis in men with non-gonococcal urethritis," Sexually Transmitted Infections, vol. 87, no. 1, pp. 38-40, 2011.

[17] R. Gdoura, W. Kchaou, C. Chaari et al., "Ureaplasma urealyticum, Ureaplasma parvum, Mycoplasma hominis and Mycoplasma genitalium infections and semen quality of infertile men," BMC Infectious Diseases, vol. 7, article 129, 2007.

[18] S. D. Mehta, C. Gaydos, I. MacLean et al., "The effect of medical male circumcision on urogenital Mycoplasma genitalium among men in Kisumu, Kenya," Sexually Transmitted Diseases, vol. 39, no. 4, pp. 276-280, 2012.

[19] V. Homfray, C. Tanton, R. F. Miller et al., "Male circumcision and STI acquisition in britain: evidence from a national probability sample survey," PLOS ONE, vol. 10, no. 6, Article ID e0130396, 2015.

[20] R. M. Gindi, K. G. Ghanem, and E. J. Erbelding, "Increases in oral and anal sexual exposure among youth attending sexually transmitted diseases clinics in Baltimore, Maryland," Journal of Adolescent Health, vol. 42, no. 3, pp. 307-308, 2008.

[21] N. Reinton, H. Moi, A. O. Olsen et al., "Anatomic distribution of Neisseria gonorrhoeae, Chlamydia trachomatis and Mycoplasma genitalium infections in men who have sex with men," Sexual Health, vol. 10, no. 3, pp. 199-203, 2013.

[22] W. Fuchs, A. Kreuter, M. Hellmich et al., "Asymptomatic anal sexually transmitted infections in HIV-positive men attending anal cancer screening," British Journal of Dermatology, 2015.

[23] R. A. Lillis, M. J. Nsuami, L. Myers, and D. H. Martin, "Utility of urine, vaginal, cervical, and rectal specimens for detection of Mycoplasma genitalium in women," Journal of Clinical Microbiology, vol. 49, no. 5, pp. 1990-1992, 2011.

[24] L. Falk, H. Fredlund, and J. S. Jensen, "Signs and symptoms of urethritis and cervicitis among women with or without Mycoplasma genitalium or Chlamydia trachomatis infection," Sexually Transmitted Infections, vol. 81, no. 1, pp. 73-78, 2005.

[25] I. Simms, K. Eastick, H. Mallinson et al., "Associations between Mycoplasma genitalium, Chlamydia trachomatis and pelvic inflammatory disease," Journal of Clinical Pathology, vol. 56, no. 8, pp. 616-618, 2003.

[26] C. L. Haggerty, P. A. Totten, S. G. Astete et al., "Failure of cefoxitin and doxycycline to eradicate endometrial Mycoplasma genitalium and the consequence for clinical cure of pelvic inflammatory disease," Sexually Transmitted Infections, vol. 84, no. 5, pp. 338-342, 2008.

[27] S. N. Taylor, "Cervicitis of unknown etiology," Current Infectious Disease Reports, vol. 16, no. 7, p. 409, 2014.

[28] L. E. Manhart, C. W. Critchlow, and K. K. Holmes, "Mucopurulent cervicitis and Mycoplasma genitalium," Journal of Infectious Diseases, vol. 190, no. 4, p. 866, 2004. 
[29] M. Jurstrand, J. S. Jensen, A. Magnuson, F. Kamwendo, and H. Fredlund, "A serological study of the role of Mycoplasma genitalium in pelvic inflammatory disease and ectopic pregnancy," Sexually Transmitted Infections, vol. 83, no. 4, pp. 319-323, 2007.

[30] A. M. Ashshi, S. A. Batwa, S. Y. Kutbi, F. A. Malibary, M. Batwa, and B. Refaat, "Prevalence of 7 sexually transmitted organisms by multiplex real-time PCR in Fallopian tube specimens collected from Saudi women with and without ectopic pregnancy," BMC Infectious Diseases, vol. 15, no. 1, article 569, 2015.

[31] S. Giakoumelou, N. Wheelhouse, K. Cuschieri, G. Entrican, S. E. M. Howie, and A. W. Horne, "The role of infection in miscarriage," Human Reproduction Update, vol. 22, no. 1, pp. 116-133, 2016.

[32] J. Hitti, P. Garcia, P. Totten, K. Paul, S. Astete, and K. K. Holmes, "Correlates of cervical Mycoplasma genitalium and risk of preterm birth among Peruvian women," Sexually Transmitted Diseases, vol. 37, no. 2, pp. 81-85, 2010.

[33] R. K. Edwards, R. J. Ferguson, L. Reyes, M. Brown, D. Theriaque, and P. Duff, "Assessing the relationship between preterm delivery and various microorganisms recovered from the lower genital tract," Journal of Maternal-Fetal and Neonatal Medicine, vol. 19, no. 6, pp. 357-363, 2006.

[34] J. Boivin, L. Bunting, J. A. Collins, and K. G. Nygren, "International estimates of infertility prevalence and treatment-seeking: potential need and demand for infertility medical care," Human Reproduction, vol. 22, no. 6, pp. 1506-1512, 2007.

[35] F. Gimenes, R. P. Souza, J. C. Bento et al., "Male infertility: a public health issue caused by sexually transmitted pathogens," Nature Reviews Urology, vol. 11, no. 12, pp. 672-687, 2014.

[36] C. Banikarim and M. R. Chacko, "Pelvic inflammatory disease in adolescents," Seminars in Pediatric Infectious Diseases, vol. 16, no. 3, pp. 175-180, 2005.

[37] A. Baczynska, P. Funch, J. Fedder, H. J. Knudsen, S. Birkelund, and G. Christiansen, "Morphology of human Fallopian tubes after infection with Mycoplasma genitalium and Mycoplasma hominis-in vitro organ culture study," Human Reproduction, vol. 22, no. 4, pp. 968-979, 2007.

[38] A. Mousavi, F. Farhadifar, R. Mirnejad, and R. Ramazanzadeh, "Detection of genital mycoplasmal infections among infertile females by multiplex PCR," Iranian Journal of Microbiology, vol. 6, no. 6, pp. 398-403, 2014.

[39] J. Grześko, M. Elias, B. Maczyńska, U. Kasprzykowska, M. Tłaczała, and M. Goluda, "Occurrence of Mycoplasma genitalium in fertile and infertile women," Fertility and Sterility, vol. 91, no. 6, pp. 2376-2380, 2009.

[40] C. Huang, H. Zhu, K. Xu, S. Wang, L. Fan, and W. Zhu, "Mycoplasma and ureaplasma infection and male infertility: a systematic review and meta-analysis," Andrology, vol. 3, no. 5, pp. 809-816, 2015.

[41] L. E. Manhart, J. S. Jensen, C. S. Bradshaw et al., "Efficacy of antimicrobial therapy for Mycoplasma genitalium infections," Clinical Infectious Diseases, vol. 61, supplement 8, pp. S802-S817, 2015.

[42] A. Lau, C. S. Bradshaw, D. Lewis et al., "The efficacy of azithromycin for the treatment of genital Mycoplasma genitalium: a systematic review and meta-analysis," Clinical Infectious Diseases, vol. 61, no. 9, pp. 1389-1399, 2015.

[43] R. H. Nijhuis, T. T. Severs, D. S. Van der Vegt, A. A. Van Zwet, and J. G. Kusters, "High levels of macrolide resistanceassociated mutations in Mycoplasma genitalium warrant antibiotic susceptibility-guided treatment," Journal of Antimicrobial Chemotherapy, vol. 70, no. 9, pp. 2515-2518, 2015.
[44] L. Falk, M. Enger, and J. S. Jensen, "Time to eradication of Mycoplasma genitalium after antibiotic treatment in men and women," Journal of Antimicrobial Chemotherapy, vol. 70, no. 11, pp. 3134-3140, 2015.

[45] L. E. Manhart, "Diagnostic and resistance testing for Mycoplasma genitalium: what will it take?" Clinical Infectious Diseases, vol. 59, no. 1, pp. 31-33, 2014.

[46] M. Unemo, K. M. Endre, and H. Moi, "Five-day azithromycin treatment regimen for Mycoplasma genitalium infection also effectively eradicates Chlamydia trachomatis," Acta DermatoVenereologica, vol. 95, no. 6, pp. 730-732, 2015.

[47] K. A. Tagg, N. J. Jeoffreys, D. L. Couldwell, J. A. Donald, and G. L. Gilbert, "Fluoroquinolone and macrolide resistanceassociated mutations in Mycoplasma genitalium," Journal of Clinical Microbiology, vol. 51, no. 7, pp. 2245-2249, 2013. 


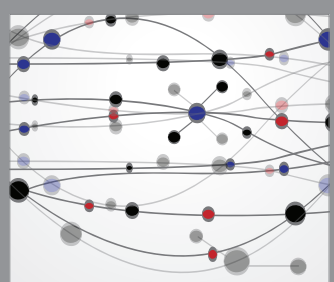

The Scientific World Journal
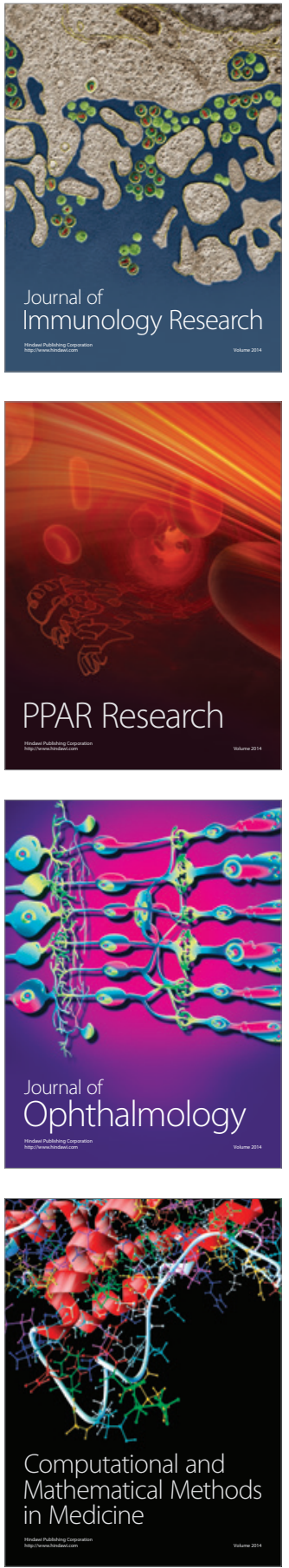

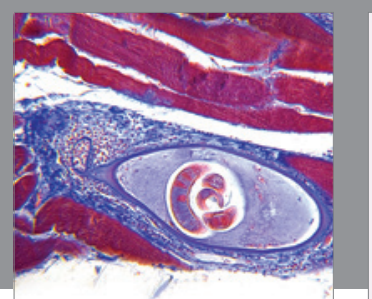

Gastroenterology Research and Practice

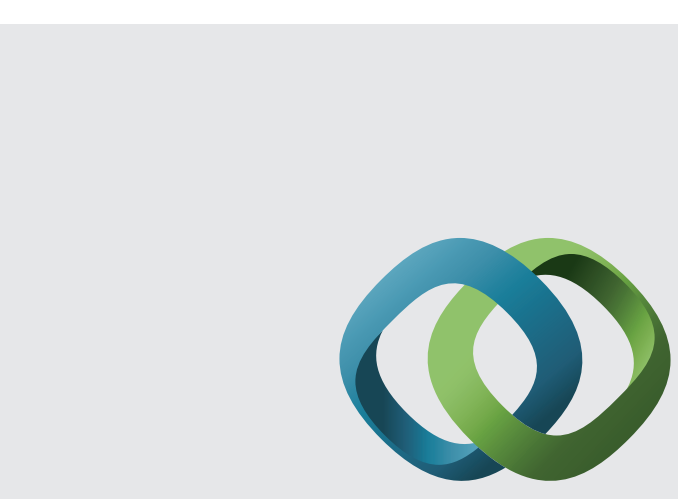

\section{Hindawi}

Submit your manuscripts at

http://www.hindawi.com
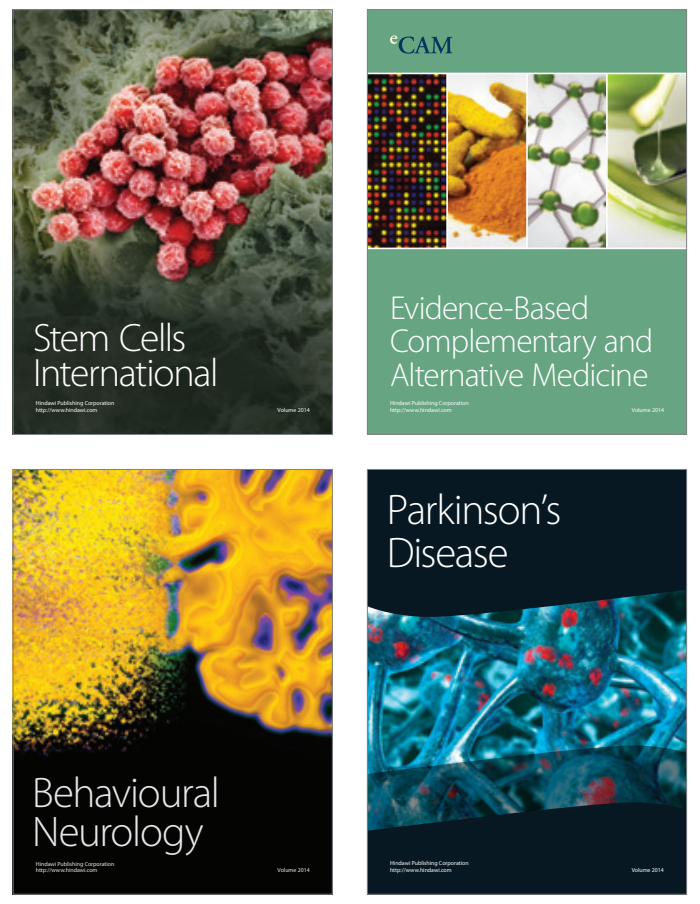
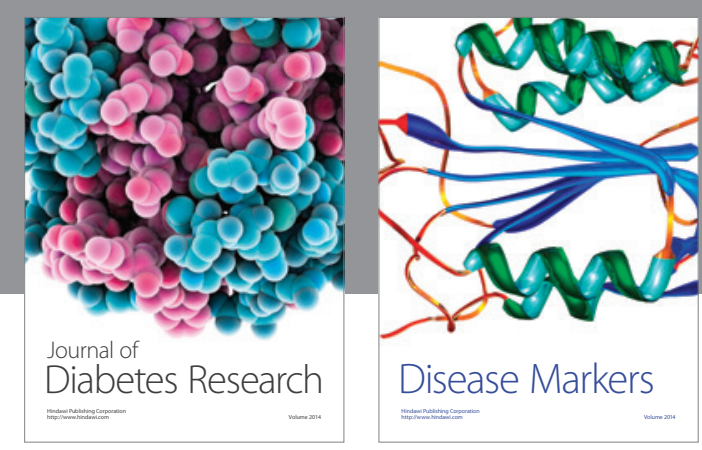

Disease Markers
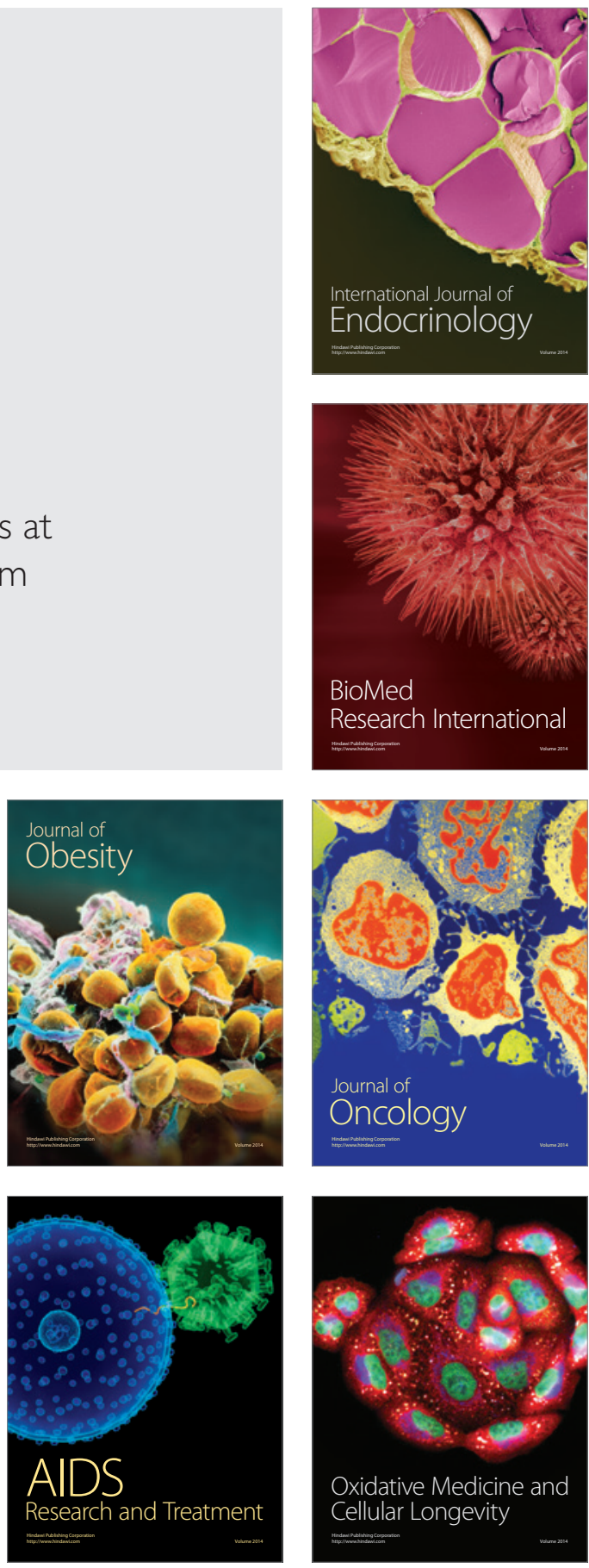